\title{
bmd: An R package for benchmark dose estimation
}

\author{
Signe M Jensen ${ }^{\text {Corresp., } 1}{ }^{,}$Felix M Kluxen ${ }^{2}$, Jens C Streibig $^{1}$, Nina Cedergreen $^{3}$, Christian Ritz $^{4}$ \\ 1 Department of Plant and Environmental Sciences, University of Copenhagen, Taastrup, Danmark \\ 2 ADAMA Deutschland GmbH, Cologne, Germany \\ 3 Department of Plant and Environmental Sciences, University of Copenhagen, Frederiksberg C, Danmark \\ 4 Department of Nutrition, Exercise and Sports, University of Copenhagen, Frederiksberg C, Danmark \\ Corresponding Author: Signe M Jensen \\ Email address: smj@plen.ku.dk
}

The benchmark dose (BMD) methodology is used to derive a hazard characterization measure for risk assessment in toxicology or ecotoxicology. The present paper's objective is to introduce the $\mathbf{R}$ extension package $b m d$, which facilitates the estimation of BMD and the benchmark dose lower limit for a wide range of dose-response models via the popular package drc. It allows using the most current statistical methods for BMD estimation, including model averaging. The package bmd can be used for BMD estimation for binomial, continuous, and count data in a simple set up or from complex hierarchical designs and is introduced using four examples. While there are other stand-alone software solutions available to estimate BMDs, the package bmd facilitates easy estimation within the established and flexible statistical environment $\mathbf{R}$. It allows the rapid implementation of available, novel, and future statistical methods and the integration of other statistical analyses. 
bmd: An $\mathbf{R}$ package for benchmark dose estimation

Signe M. Jensen ${ }^{1}$, Felix M. Kluxen², Jens C. Streibig ${ }^{1}$, Nina Cedergreen ${ }^{3}$, Christian Ritz ${ }^{4}$

${ }^{1}$ Department of Plant and Environmental Sciences, University of Copenhagen, Taastrup,

Denmark

${ }^{2}$ ADAMA Deutschland GmbH, Cologne, Germany

${ }^{3}$ Department of Plant and Environmental Sciences, University of Copenhagen, Frederiksberg C, Denmark

${ }^{4}$ Department of Nutrition, Exercise and Sports, University of Copenhagen, Frederiksberg C, Denmark

Corresponding author: Signe Marie Jensen ${ }^{1}$

Email address: smj@plen.ku.dk

\section{ABSTRACT}

2 The benchmark dose (BMD) methodology is used to derive a hazard characterization measure

3 for risk assessment in toxicology or ecotoxicology. The present paper's objective is to introduce

4 the $\mathbf{R}$ extension package $b m d$, which facilitates the estimation of BMD and the benchmark dose

5 lower limit for a wide range of dose-response models via the popular package $d r c$. It allows

6 using the most current statistical methods for BMD estimation, including model averaging. The

7 package bmd can be used for BMD estimation for binomial, continuous, and count data in a

8 simple set up or from complex hierarchical designs and is introduced using four examples. While

9 there are other stand-alone software solutions available to estimate BMDs, the package $b m d$ 
10 facilitates easy estimation within the established and flexible statistical environment $\mathbf{R}$. It allows

11 the rapid implementation of available, novel, and future statistical methods and the integration of

12 other statistical analyses.

\section{INTRODUCTION}

Risk assessment in human toxicology and ecotoxicology quantifies the relationship between the exposure to a chemical (or other stressor) and a hazard characterization measure of the chemical.

If the exposure dose/concentration is estimated to be less than the dose/concentration estimated to have adverse effects (the point of departure (POD)) then the risk associated with exposure to the chemical agent is considered acceptable. In practice, the POD is often modified by safety or uncertainty factors to account for inter-species differences and inter- and intra-individual differences in the target population. This modified value is usually called the reference dose/concentration or limit value, depending on the context. The two main approaches for deriving PODs are the No Observed Adverse Effect Level (NOAEL) and the Benchmark Dose (BMD) methodology. Note that for concentrations also NOAEC (No Observed Adverse Effect Concentration) and less commonly BMC (Benchmark Concentration) are used, as is NOEL, (No Observed Effect Level), NOED (No Observed Effect Dose) or NOEC, (No Observed Effect Concentration), in the context where any effect is considered adverse. Whether level, dose, or concentration is used depends on how the test organism/system is exposed to the chemical agent, but in the context of developing methods for benchmark dose estimation the term dose will be used to quantify exposure.

The NOAEL is the highest dose that does not lead to an observable adverse change in the investigated response. Hypothesis testing usually complements the Lowest Adverse Effect Level 
33 (LOAEL) determination, i.e., the next dose level above the NOAEL. The NOAEL will be an

34 experimentally tested dose and it depends strongly on the dose spacing. Poor experimental

35 design, high variation, and low statistical power lead to relatively higher NOAELs, which is

36 problematic from a conservative point of view and can only be mitigated by toxicological

37 weight-of-evidence assessment, considering multiple studies or endpoints within a single study

38 or by means of additional safety factors.

The BMD approach aims to reduce some of these issues associated with NOAEL derivation and

40 relies on dose-response modelling. It is, therefore, less dependent on the tested doses. The BMD

methodology was initially introduced by Crump (Crump, 1984) for binomial response data, but

has subsequently been extended with definitions for continuous response data (Gaylor \& Slikker,

1990; Crump, 1995; Budtz-Jørgensen, Keiding \& Grandjean, 2001). The BMD methodology is

now recommended by OECD and other regulatory authorities (Davis, Gift \& Zhao, 2011;

Organisation for Economic Co-operation and Development (OECD), 2012a; Hardy et al., 2017).

The BMD is defined as the dose associated with a predefined small change in the response, the

47 benchmark response (BMR), from the background response level. This is a key difference to the

NOAEL, which refers to a level with no observable effect, which may be biased by statistical

power, or experimental sensitivity (Brescia, 2020).

BMRs of 5 and $10 \%$ are often used; however, appropriate BMRs for different endpoints need to be discussed within the (eco)toxicological community (Slob, 2017; Haber et al., 2018; Jensen, Kluxen \& Ritz, 2019; Kluxen, 2020), as they are not scientifically reasoned, which is similar to the use of $\mathrm{p}<0.05$ (Salsburg, 2001; Kennedy-Shaffer, 2019). BMDs are essentially similar to

54 effective doses or concentrations but often located in more data-sparse regions of the doseresponse curve. In practice, reference values or limit values are derived from the BMD lower 
56 limit (BMDL), which is defined as the lower limit of a one-sided confidence interval of the BMD

57 estimate (see, Fig. 1).

58 Currently, mainly two comprehensive software packages are available for BMD analysis: BMDS

59 developed by US Environmental Protection Agency (EPA) (Davis, Gift \& Zhao, 2011) and

60 PROAST developed by the Netherlands National Institute for Public Health and the Environment

61 (RIVM) in collaboration with Open Analytics (Varewyck \& Verbeke, 2017; Slob, 2018). The

62 two packages differ in several ways. BMDS includes several dose-response models, but it (for

63 now) only includes Bayesian model averaging and for binary data only. In contrast, PROAST

64 offers model averaging for both binary and continuous response data but includes fewer dose-

65 response models. The two packages also differ in how BMDL is derived. A few other more

66 specialized packages have also been developed for BMD analysis (Yang, Allen \& Thomas,

67 2007; Wheeler \& Bailer, 2008; Shao \& Shapiro, 2018).

68 Over the past 25 years, the open-source environment $\mathbf{R}$ ( $\mathrm{R}$ Core Team, 2018) has developed into

69 an extremely powerful statistical software where many extension packages offer specialized

70 functionality. One such extension package is $d r c$ developed for the analysis of dose-response

71 data (Ritz et al., 2019).

72 The present paper's objective is to introduce the $\mathbf{R}$ extension package $b m d$ and help

73 (eco)toxicologists with using the tool by the evaluation of multiple case studies and simple

74 example code. The package allows using the most current statistical methods for BMD

estimation in R. It can be seen as a flexible alternative to BMDS and PROAST in terms of

76 available models, a higher degree of user-control over models, and more approaches for BMDL

77 estimation and model averaging. The option of using sandwich variance-covariance estimates to

78 adjust for model misspecifications is an important addition. The $\mathbf{R}$ package also offers the 
79

80

81

82

83

84

85

86

87

possibility to work with count data. An option not available in any other BMD software. The other clear distinction of $b m d$ is that it can be used within the $\mathbf{R}$ environment and thus supplement other statistical analyses within the same software. Further, other extensions that are or will be developed for $\mathbf{R}$ can be directly used together with $b m d$. This flexibility is currently not achieved in other BMD software.

\section{BMD ESTIMATION}

Estimating a BMD and its corresponding BMDL depends on the type of response data considered, the definition used for defining BMD, the predefined BMR, the choice of doseresponse model, and the approach used for estimating BMDL. The dose-response model is fitted to data using $d r c$. Since $d r c$ uses powerful self-starter functions, the fitting process is automated. The fit can then be visually assessed, and the distribution of the residuals investigated. The fit can also be compared to other candidate models, and model averaging may be performed. Below, the individual steps of the process are described.

\subsection{Defining BMD for a predefined BMR}

The BMD is defined as the dose resulting in a pre-specified small change, denoted the BMR, in the response relative to the control group's response level. In practice, defining BMD and specifying BMR goes hand in hand (Jensen, Kluxen \& Ritz, 2019). Different types of changes in the response may be relevant, i.e., absolute versus relative changes compared to the control response level, and accordingly, different definitions of the BMD exist. However, not all definitions are relevant to all types of endpoints. The definitions available in the package $b m d$ are 
101 described below in detail and are summarized in Table S1 in the supplementary material. The

102 definitions below are for increasing dose-response curves; for decreasing dose-response curves,

103 the same principles apply, but with a slight change in the definitions (for example, the definition

104 of additional risk for decreasing dose-response curves is: $\left.B M R=p_{0}-f(B M D, \beta)\right)$.

105

106

107

108

109

Excess risk

$$
B M R=\frac{f(B M D, \beta)-p_{0}}{1-p_{0}}
$$

$$
B M R=f(B M D, \beta)-p_{0}
$$

$f$ denotes the dose-response model, and $\beta$ is the vector of parameters in the model. $p_{0}$ represents

the mean background response level, i.e., the probability of an adverse effect. The background response level is typically estimated from the model as $p_{0}=\lim _{\operatorname{dos} e \rightarrow 0} f($ dose, $\beta)$ but may also be estimated from a control group or be specified based on prior knowledge (Jensen, Kluxen \&

114 Ritz, 2019). Notice that if the background response level, $p_{0}$, equals 0 , the two definitions, 115 additional risk (Eq. 1), and excess risk (Eq. 2), are identical. Excess risk is the most common definition used for binomial response data. The excess risk definition (Eq. 2) is recommended by

117 both the US EPA and the European Food Safety Authority (EFSA) (US Environmental

118 Protection Agency, 2012; Hardy et al., 2017). Appropriate BMR values should ideally be based 119 on expert biological or toxicological knowledge about the test system, but in practice, the 
120 decision is often driven by statistical considerations (Jensen, Kluxen \& Ritz, 2019). US EPA and

121 EFSA recommend choosing a BMR in the lower end of the specific data set's observable dose

122 range. A BMR of $10 \%$ using the excess risk definition is often within the dose-range and a

123 common choice for reporting results of a BMD analysis with binomial data (US Environmental

124 Protection Agency, 2012; Hardy et al., 2017; Jensen, Kluxen \& Ritz, 2019).

125 2.1.2 Continuous response data

126 For continuous response data, the analogous definitions of additional and excess risk are based

127 on the so-called hybrid approach (Gaylor \& Slikker, 1990; Budtz-Jørgensen, Keiding \&

128 Grandjean, 2001, Jensen, Kluxen \& Ritz, 2019). As continuous data typically reflects a response

129 and not an adverse effect, the hybrid approach "transform" the continuous response

130 measurements to the probability of observing an adverse effect by using a cut-off. The hybrid

131 approach is defined as in e.g., Jensen, Kluxen \& Ritz, 2019. Specifically, the hybrid approach

132 defines BMD as the dose corresponding to a predefined increase (BMR) in the probability of the

133 response values falling below (or exceeding) a certain cut-off value on the response scale, i.e.,

134 the cut-off divides the continuous response scale into normal and abnormal values. In contrast to

135 binomial data, the background response level, $p_{0}$, of abnormal responses is not directly given by

136 the data but has to be specified using a pre-specified cut-off, possibly based on historical data

137 (Jensen, Kluxen \& Ritz, 2019). Assuming a normal distribution for the unexposed (background)

138 population the background probability of an adverse event, $p_{0}$, may be specified as

$$
p_{0}=1-\Phi\left(\frac{x_{0}-f(0, \beta)}{\sigma}\right)
$$


140 where $x_{0}$ is the organism response level considered to be adverse, $\Phi$ denotes the cumulative

141 distribution function for a standard normal distribution, and $\sigma$ is the standard deviation for the

142 control group. With this definition of the background response level (Eq. 3), the hybrid approach

143 leads to the definition of the BMD as the dose solving the following equations:

145 Hybrid approach (additional definition)

$$
\mathrm{BMR}=1-\Phi\left(\frac{x_{0}-f(\mathrm{BMD}, \beta)}{\sigma}\right)-p_{0}
$$

147 Hybrid approach (excess definition)

$$
\mathrm{BMR}=\frac{1-\Phi\left(\frac{x_{0}-f(\mathrm{BMD}, \beta)}{\sigma}\right)-p_{0}}{1-p_{0}}
$$

149 For some outcomes, it may be a "natural" choice to choose a specific level, $x_{0}$, on the response 150 scale to define the cut-off for adverse response levels. However, the most common case is to use 151 a cut-off in terms of a pre-specified number of standard deviations, circumventing the need to

152 decide on an absolute level. For example, US EPA recommends using a cut-off of 1 standard 153 deviation (US Environmental Protection Agency, 2012). Both options for defining the

154 background level are available in $b m d$. When using the hybrid approach (Eq. 4), typical values of 155 BMR are 5\% or 10\% (US Environmental Protection Agency, 2012). 
156

157 Additional definitions that are also used in the literature were included in $b m d$ available for both 159 2017):

160

Added response

$$
\mathrm{BMR}=f(\mathrm{BMD}, \beta)-f(0, \beta)
$$

161 Extra response

$$
\mathrm{BMR}=\frac{f(\mathrm{BMD}, \beta)-f(0, \beta)}{f(\infty, \beta)-f(0, \beta)}
$$

Relative response

$$
\mathrm{BMR}=\frac{f(\mathrm{BMD}, \beta)-f(0, \beta)}{f(0, \beta)}
$$

163

164 In definitions (7)-(9), BMR is defined as an absolute or relative change in the response from the background response level $f(0, \beta)$, as estimated by the model. The added response definition expression mathematically corresponds to the additional risk definition for binomial data where the response is an adverse event. Suppose the upper limit is 1 (for increasing dose-response models or the lower limit is 0 for decreasing dose-response relationships). In that case, the extra response definition corresponds mathematically to the excess risk definition for binomial data.

170 The relative response definition (Eq. 9) is sometimes referred to as deriving the critical effect 171 size (Slob, 2017). US EPA recommends reporting the critical effect size and results from the 172 hybrid approach using BMR values of 5\% or 10\% as appropriate (US Environmental Protection 
173 Agency, 2012). EFSA recommends reporting the critical effect size with the default BMR value

174 of $5 \%$ (Hardy et al., 2017).

175 2.1.4 All types of response data

176 Finally, it is an option in bmd to specify directly the response level for which to find the dose.

177 Directly defined

178

$$
\mathrm{BMR}=f(\mathrm{BMD}, \beta)
$$

\subsection{Estimation}

All types of BMD estimates are calculated in an after-fitting step when using the package $b m d$.

After-fitting refers to deriving estimates and corresponding standard errors for parameters that do not directly enter the model parameterization and hence have to be estimated in a subsequent step using the delta method. The BMD estimating function in $b m d$ is called bmd( $)$ and takes a $d r c$ object (model fit) as its first argument. The alternative to after-fitting would be to reparameterize the model to include the BMD of interest as a model parameter. The after-fitting approach has, however, the advantage that it suffices to fit the dose-response model once in a

187 parameterization that has proven to be the most robust for estimation (Ritz et al., 2015). Accordingly, after-fitting provides an improvement over the sometimes quite unstable reparameterization approach. This is especially the case when estimating a BMD corresponding to a very low BMR value, which may then be in an area with little or no information in data.

\subsection{Choice of dose-response model}

192 A key distinguishing feature of $b m d$ is that it inherits flexibility in available models from the 193 package $d r c$ (Ritz et al., 2019). Among others, the parametric dose-response models available 
194 include the well-known and often used multi-stage, log-logistic and Weibull models and the

195 flexible class of fractional log-logistic models proposed by Namata et al. (Namata et al., 2008).

196 As a non-parametric alternative, the function bmdiso() monotonizes the sequence of response

197 values based on the pool-adjacent-violators algorithm. Based on the monotonized sequence,

198 linear interpolating splines are used to build an isotonic regression model as the dose-response

199 model used for BMD estimation (Piegorsch et al., 2012, 2014; Lin, Piegorsch \& Bhattacharya,

200 2015). Table S2 lists all models from $d r c$ available for BMD estimation in $b m d$. All the models

201 shown are available for binomial, continuous, and count response data. Even more model

202 expressions may be estimated by fixing one or more parameters. We refer to Ritz, Jensen, et al.

203 (2019) for more details.

\subsection{Defining BMDL}

206

207

208

209

210

211

212

213

214

215

216

Different approaches for estimating the BMDL have been proposed. They can be summarized as three general types; asymptotic approaches, inverse regression and based on bootstrapping. Among the asymptotic approaches, the Wald-type confidence intervals are the most simple. The one-sided Wald-type interval needed to estimate the BMDL is found by combining information on the parameters included in the model by using the delta method. The Wald-type intervals may result in unrealistic negative BMDL estimates, which in practice will be truncated at 0 because of the symmetric confidence interval. Especially in the low dose area, this may be a problematic assumption. One way to work around this is to use a transformation (typically the logarithm) to avoid negative values. Inverse regression reports the BMDL as the dose associated with the upper limit of the confidence band for the change in response reaching the predefined BMR (Buckley, Piegorsch \& West, 2009; Fang, Piegorsch \& Barnes, 2015). 
217 Bootstrap methods for estimating BMDL may rely on non-parametric, parametric, and semi-

218 parametric strategies depending on the type of response data. The non-parametric bootstrap is

219 based on resampling with replacement from each dose group of the original data set. The

220 parametric bootstrap for continuous data samples from a normal distribution with dose-specific

221 mean but equal standard deviation, assuming equal variance between groups, i.e., response

222 values are sampled from:

223

$$
\overline{Y_{i j}} \approx N\left(E\left(Y_{i}\right), S D\left(Y_{0}\right)\right)
$$

224 for observation $j$ in dose-group $i$. In case of binomial data, each bootstrap data set is sampled

225 from a binomial distribution with dose-specific numbers of observations and probability of an

226 event. That is, response values are sampled from:

$$
\overline{Y_{i j}} \approx \operatorname{Binom}\left(N_{i}, \frac{Y_{i}}{N_{i}}\right)
$$

228 where $\frac{Y_{i}}{N_{i}}$ denotes the observed proportion of the population in dose group $i$ experiencing the 229 event.

230 In case a dose has only non-events or only events, shrinkage is used to avoid that the resampling 231 always produces 0 or 1 , respectively. In this case, data is sampled from

$$
\overline{Y_{i j}} \approx \operatorname{Binom}\left(N_{i}, \frac{Y_{i}+1 / 4}{N_{i}+1 / 2}\right)
$$
as suggested elsewhere (Piegorsch et al., 2012).

234 Semi-parametric bootstrapping, only available for continuous response data, is done by sampling 235 with replacement from the residuals assuming exchangeability over all observations. 


\subsection{Exploring model assumptions}

238 For continuous data, residual plots showing standardized residuals against predicted values can

239 be used to assess variance homogeneity. A random scatter plot will support the model

240 assumptions whereas clear patterns in the plot indicate deviations from model assumptions.

241 Assessment of the normality assumption can be based on quantile-quantile plots on the residuals.

242 For an example on how to do a visual assessment of model assumptions, we refer to Ritz, Jensen,

243 et al., 2019. Using the visual assessment of residual and quantile-quantile plots is associated with

244 some degree of subjective evaluation. However, the test-based assessment also has limitations, as

245 it might result in overfitting, or because the assumptions underlying the tests themselves are not

246 fulfilled (Wang \& Riffel, 2011).

247 Assessment of over-dispersion for binomial or count data can be done by comparing residual

248 deviance to degrees of freedom. If the residual deviance is higher than the degrees of freedom,

249 there is excess variation in data (over-dispersion) not accounted for by the model. Alternatively,

250 a model allowing an extra variance parameter, i.e., a negative binomial model can be fitted and

251 the need for the extra parameter evaluated by comparing models with and without the extra

252 parameter, for example, using AIC (Akaike information criteria). Finally, the model may be

253 estimated using sandwich estimates (see section 2.6). A large change in the standard errors

254 indicate over-dispersion in data. 


\section{2.6 Dealing with distributional misspecification}

257 Extreme or, by other means, deviating observations may be handled already in the model-fitting

258 step using robust estimation in $d r c$. The resulting variance-covariance matrix will be propagated

259 to the subsequent after-fitting step where BMD and BMDL are derived.

260 When assumptions regarding normality and/or the variance homogeneity are not satisfied,

261 consistent estimates of the standard errors can be obtained by adjusting the estimated variance-

262 covariance matrix. One way of doing this is to use a modified variance-covariance matrix

263 defined as:

264

$$
\operatorname{var}(\hat{\beta})=\hat{A}^{-1} \hat{B}\left(\hat{A}^{-1}\right)^{T}
$$

265 where $\hat{A}$ is the usual estimate of the variance based on the information matrix, and $\hat{B}$ is a

266 correction term based on the first derivatives on the log-likelihood function. Due to the form of

267 the modified variance-covariance matrix, the resulting adjusted standard errors are referred to as

268 sandwich estimates. Notice that the sandwich approach only modifies standard errors but leaves

269 estimated model parameters unaltered. Accordingly, the underlying assumption of a correctly

270 specified mean function remains.

271 Finally, log-normal distributed data may be handled by log-transforming the response variable

272 before fitting the dose-response model, which leads to a change in the interpretation of BMD and

273 BMDL, now relating to a pre-determined change in the response on log-scale. Alternatively, the

274 transform-both-sides approach could be used such that the assumed dose-response relationship,

275 and accordingly, BMD and BMDL will retain their original interpretation (Carroll \& Ruppert, 276 1988).

277 


\section{$278 \quad$ 2.7 Model averaging}

279 Interpolation in a dose region with little or no data, as is typically the case for BMD analysis, is

280 highly dependent on the fit and choice of dose-response model. To partly overcome this issue, it

281 has been proposed to evaluate several models and subsequently select the best model determined

282 by means of some goodness-of-fit criterion (Slob, 2002). However, the uncertainty pertaining to

283 the model selection process is not incorporated in the BMD and the associated BMDL. Indeed,

284 results based on a best fitting model found using a model selection procedure may result in

285 biased estimates of BMD and non-protecting estimates of BMDL, i.e., too high BMDL estimates

286 with coverage below the nominal level (West et al., 2012; Ringblom, Johanson \& Öberg, 2014).

287 As a consequence, model averaging has been repeatedly advocated for BMD analysis by several

288 authors (e.g., Faes et al. 2007; Jensen and Ritz 2015; Kang, Kodell, and Chen 2000; Namata et

289 al. 2008; Wheeler and Bailer 2007, 2008) as well as regulating authorities (Hardy et al., 2017).

290 The use of model averaging has the consequence that the flexibility of the assumed candidate

291 models to some extent replaces lack of data. The package $b m d$ offers a large set of dose-response

292 models and accordingly a high flexibility of the candidate set of models for model averaging.

293 Another advantage is that it allows for an automated approach once the candidate models are

294 specified.

295 Two different approaches may be used for estimating BMD by model averaging. One approach

296 is to make a weighted average of the BMD estimates from all the candidate models (e.g., Bailer,

297 Noble, and Wheeler 2005; Kang, Kodell, and Chen 2000; Moon et al. 2005; Namata et al. 2008).

298 The other is to average entire curves and then find the BMD as the dose associated with the

299 change of interest in the response generated by this weighted averaged model, i.e., using the 
300 appropriate definition (Wheeler \& Bailer, 2007, 2008). Weights are usually based on some

301 measure of goodness of fit. A common choice is the AIC weights defined as:

$$
w_{k}=\frac{\exp \left(\frac{-\Delta_{k}}{2}\right)}{\sum_{i=1}^{K} \exp \left(\frac{-\Delta_{i}}{2}\right)}
$$

302

303 Here $K$ denotes the total number of candidate models and $\Delta_{k}=\mathrm{AIC}_{k}-\underset{i}{\min } \mathrm{AIC}_{i}$ is the AIC-

304 difference for model $k$. Alternatively, weights based on the Bayesian information criteria (BIC)

305 (Claeskens \& Hjort, 2008) or user-defined weights may be provided.

306 Estimating the BMDL corresponding to a model-averaged BMD is less straightforward and has

307 accordingly been the subject of much methodological research (Jensen, Kluxen \& Ritz, 2019).

308 Three different approaches are available in $b m d$.

309 The first option is using a one-sided Wald confidence intervals with standard error approximated 310 by a variance inequality by Buckland et al. (Buckland, Burnham \& Augustin, 1997):

311

$$
\operatorname{var}\left(\mathrm{BMD}_{M A}\right) \leq\left(\sum_{k=1}^{K} w_{k} \cdot \sqrt{\operatorname{var}\left(\mathrm{BMD}_{k}\right)+\left(\mathrm{BMD}_{k}-\mathrm{BMD}_{M A}\right)^{2}}\right)^{2}
$$

312 Here, $B M D_{k}$ is the BMD for model $\mathrm{k}$ and $B M D_{M A}$ is the weighted average of BMD estimates.

313 The second option is using a weighted average of the BMDLs from all the candidate models, in 314 the same way, BMD estimates are averaged to get the model-averaged BMD (Kang, Kodell \& 315 Chen, 2000): 


$$
\mathrm{BMDL}_{M A}=\sum_{k=1}^{K} w_{k} \cdot \mathrm{BMDL}_{k}
$$

317 With weights, $w_{k}$, specified as above, e.g., using Eq. 10. Finally, different bootstrap approaches

318 may be used to estimate BMDL corresponding to the model-averaged BMD. For model

319 averaging based on entire curves, the only option for estimating the BMDL is to use bootstrap.

\subsection{Hierarchical designs}

Data obtained from designs with a hierarchical or nested structure do not comply with the basic assumptions of independence underlying the classical dose-response regression analysis. Hence, sticking to these models may result in biased BMDL estimates, i.e., too high or low BMDL estimates with coverage far from the nominal level, usually 95\%. Preferably, a mixed effects dose-response model should be used to model such data in order to take into account the correlation structure in data (Ritz, Gerhard \& Hothorn, 2013). An alternative approach could be to adjust the estimated standard errors of BMD using sandwich estimates. Another alternative is to fit dose-response models for each independent subsample, and combine the estimates using a meta-analytic approach (Ritz et al., 2019). This latter approach has been shown to result in estimates with stable and near nominal coverage for the closely related effective doses (Jiang \& Kopp-Schneider, 2014), and it can therefore be used as an attractive alternative to the more complex fitting of mixed effects dose-response models. An example of this meta-analytic approach is provided in Example 3.4. A similar two-step meta-analytic approach could be used to integrate historical data (Ritz et al., 2019; Jensen, Kluxen \& Ritz, 2019). 
337

338

339

340

341

342

343

344

345

346

347

348

349

350

351

352

353

354

355

356

357

358

\section{EXAMPLES}

The $\mathbf{R}$ package $b m d$ builds on the flexibility of $d r c$, and thereby facilitates BMD and BMDL estimation for a wide range of dose-response models. It was first mentioned as a function in Ritz et al. (2015) but has been substantially updated since 2015 with the one common denominator being that both versions utilizes the functionality available in $d r c$. The main function in the package $b m d$ is bmd(), which uses a $d r c$ object (a dose-response model fit) and can be easily extended by including arguments. In the following, we revisit four data examples from the literature. $\mathbf{R}$ code for the different examples is provided as supplementary material. In short, after installation of the $\mathbf{R}$ package from GitHub, the basic approach is to fit a curve using $\operatorname{drm}()$.

$\operatorname{library}(\mathrm{drc})$

modelfit $<-\operatorname{drm}(\ldots)$

and then apply the function bmd()

library(bmd)

$\operatorname{bmd}(\operatorname{modelfit}, \mathrm{bmr}=\ldots$, backgType $=$ “...”, def $=$ “...”)

with a specification of BMR, the background type (usually "modelbased"), and the type of definition as indicated in Table S1.

\subsection{Binomial data: An earthworm toxicity test with chloroacetamide}

Our first example revisits data from an earthworm toxicity test (Hoekstra, 1987). For each of six concentrations (including a zero control), 40 earthworms were exposed to the herbicide chloroacetamide, and the resulting number of dead earthworms was counted. The control or 
359 natural mortality was 3/40. Following the authors, we fitted a three-parameter log-normal model

360 to the data, estimating the natural mortality as a lower limit. Figure 1 shows the resulting model

361 fit. The model-based estimated natural mortality was 0.10 (95\% CI: $0.03-0.17)$.

362 In this example, we considered a BMR of 5\%, as this small change in probability of an adverse

363 event (death) was well covered by the dose-range. The BMD corresponding to a BMR of 5\%

364 using the additional risk definition was the dose associated with a proportion of dead earthworms 365 equal to

$$
\mathrm{BMR}+p_{0}=0.10+0.05=0.15
$$

367 The resulting estimated BMD and BMDL were $20.02 \mathrm{mg} / \mathrm{kg}$ and $15.40 \mathrm{mg} / \mathrm{kg}$, respectively. The

368

369

370

371

372

373

374

375

376

377

378

379

380

381

BMD corresponding to a BMR of 5\% using the excess risk definition was the dose associated with a proportion of dead earthworms equal to

$$
\operatorname{BMR} \cdot\left(1-p_{0}\right)+p_{0}=0.05 \cdot(1-0.10)+0.10=0.145
$$

The resulting estimated BMD and BMDL for the excess risk definition were $19.79 \mathrm{mg} / \mathrm{kg}$ and $15.15 \mathrm{mg} / \mathrm{kg}$, respectively.

\subsection{Count data: A toxicity test in aquatic plants}

As part of an experiment examining the toxicity of metsulfuron-methyl on different aquatic plant species, Cedergreen et al. (2004) considered the effect on Elodea canadensis. Vegetative shoots were placed in an aquarium growth cabinet with a photoperiod of $16 \mathrm{~h}$ and at day/night water temperatures of $18 / 15^{\circ} \mathrm{C}$. Six E. canadensis shoots were exposed to each of seven different concentrations of metsulfon-methyl $(0,0.01,0.1,1,10,100,1000 \mu \mathrm{g} / \mathrm{L}$ medium $)$ in an artificial nutrient medium. Plants were harvested after 14 days and lateral shoots counted among other endpoints. 
382 A three-parameter log-logistic model was fitted to the shoot counts assuming Poisson distributed

383 data. Figure S1 in the supplementary material shows the resulting model fit. The figure also

384 shows all data points making it clear that data contains several 0 s and also other ties. The fitted

385 model described data adequately, as also indicated by a residual plot (shown in the $\mathbf{R}$ code in the 386 supplementary material).

387 We considered an absolute change in the response of one less shoot for these data, as biological 388 implications on 1 less shoot can be considered relevant. That is, BMD was estimated with the 389 added definition and a BMR of 1 shoot. Using nonparametric bootstrap, we found BMD equal to $390 \quad 0.133 \mu \mathrm{g} / \mathrm{L}$ and BMDL equal to $0.052 \mu \mathrm{g} / \mathrm{L}$.

391 If we ignored that these data were count data and instead carried out the analysis assuming a normal distribution, the resulting BMD and BMDL would be $0.254 \mu \mathrm{g} / \mathrm{L}$ and $0.092 \mu \mathrm{g} / \mathrm{L}$, respectively. Ignoring that these were count data would result in less protective estimates in terms of a much higher BMD and a somewhat higher BMDL that was based on much wider confidence intervals.

\subsection{Count data: A toxicity test with copper under varying temperature}

Cedergreen et al. (2016) examined the effect of varying temperature on the toxicity of copper on the nematode Caenorhabditis elegans (Maupas). As part of the experiment, 12 nematode worms were exposed to each of five copper concentrations $(1,3,8,20,40 \mathrm{mg} / \mathrm{L}$ agar) under varying temperatures with daily fluctuations of \pm 4 degrees around a mean temperature of $20^{\circ} \mathrm{C}$. Besides, 36 worms were exposed to the same temperature fluctuations but were considered controls with no copper exposure. During the experiment's reproduction phase, the nematodes were moved to 
405 lifespan of each nematode. The number of fertile eggs and hatched juveniles were counted as 406 offspring.

407 We followed the guidelines for continuous data from US EPA and estimated the BMD associated 408 with a BMR of $10 \%$ using the relative definition, i.e., the concentration associated with a $10 \%$ 409 reduction in the total number of offspring relative to the control group. A three-parameter log410 logistic model was fitted to data under the assumption of Poisson distributed data. Figure S2 411 shows the resulting model fit. The resulting BMD and BMDL were $10.47 \mathrm{mg} / \mathrm{L}$ agar and 9.40 $412 \mathrm{mg} / \mathrm{L}$ agar, respectively.

413 It may be argued that the above model is problematic due to premature mortality (Delignette414 Muller et al., 2014). An alternative would accordingly be to use a weighted dose-response model 415 taking into account the lifespan of the nematodes. The BMD from this new model should now be 416 interpreted as the concentration associated with a $10 \%$ change in the number of offspring per day 417 (the lifespan unit) relative to the control group. Because of the two different interpretations, the 418 BMD and BMDL estimates from this model $(31.16 \mathrm{mg} / \mathrm{L}$ and $28.89 \mathrm{mg} / \mathrm{L}$, respectively) cannot 419 be directly compared to those from the first model.

420 A second issue related to reproduction data is that count data often exhibit overdispersion, i.e., 421 more variation than can be explained by the model. Two different approaches can be used to 422 overcome this challenge; one is to change the underlying assumed distribution, the other to 423 adjust the standard errors. The first option would, for example, be to use a negative binomial 424 distribution instead of the common choice of the Poisson distribution. The resulting BMD and 425 BMDL were then $27.99 \mathrm{mg} / \mathrm{L}$ and $15.99 \mathrm{mg} / \mathrm{L}$, respectively. The second option is to use 426 sandwich estimates when estimating the BMDL while trusting that the dose-response model 427 function appropriately describes the mean trend in data. The result of this approach was a BMD 
428 of $31.16 \mathrm{mg} / \mathrm{L}$ and a BMDL of $18.63 \mathrm{mg} / \mathrm{L}$. Notice that both procedures accounting for

429 overdispersion resulted in a large decrease of the BMDL, indicating that overdispersion was

430 indeed present in these data.

431

4323.4 Continuous data: A fish test

433 OECD describe data from a 21 day fish test following the guidelines by OECD GL204 and

434 using the test organism Rainbow trout Oncorhynchus mykiss (Organisation for Economic Co-

435 operation and Development (OECD), 2006). The Rainbow trouts were held in $14-15{ }^{\circ} \mathrm{C}$ water

436 and exposed to one of 7 concentrations (6 nonzero concentrations + control) of an unknown

437 agent. After 28 days, the wet weight was registered. There were ten replicates per concentration.

438 However, for some higher doses, missing values occurred, resulting in a final data set with only

43961 observations of the weight. Ignoring such missing values may lead to biased results,

440 depending on the mechanism causing the missing values, but we will ignore the potential

441 problem here for illustrating the BMD methodology.

442 Following OECD (2006), we fitted a two-parameter exponential decay model to the Rainbow

443 trout data. Figure 2 (full line) reveals an appropriate fit to data supported by residual and QQ

444 plots (see supplementary material: $\mathbf{R}$ code for the examples).

445 For a BMR of 5\% using the hybrid approach with 2 standard deviations as the cut-off the BMD

446 and BMDL were $4.23 \mathrm{mg} / \mathrm{L}$ and $2.13 \mathrm{mg} / \mathrm{L}$, respectively.

447 While the two-parameter exponential decay model showed a good fit to data, other models may

448 give a similar fit to data. The three-parameter log-logistic and two different three-parameter

449 Weibull models showed similar reasonable visual fit to data (see Fig. 2). Comparing the models 
450 using AIC also showed a similar fit of the four models (Table 1). The computation time (using a

451 standard Dell laptop) for 1000 bootstrap samples using the four models was app. 3 min for the

452 estimate averaging and app. $5 \mathrm{~min}$ for the curve averaging. For comparison, the computation

453 time for this data set using PROAST (web application) was app. 6 min for model averaging

454 based on four models and 1000 bootstrap samples.

455 Model averaging based on the four models using non-parametric bootstrap for estimating BMDL

456 resulted in a BMD much higher and a BMDL rather close to the corresponding estimates from

457 the original (exponential decay) model. The alternative model averaging approach where entire

458 curves were averaged before finding BMD and BMDL resulted in a slightly higher BMD but a

459 slightly lower BMDL.

\subsection{Binomial data in a hierarchical design: An acute toxicity test with $\alpha$-cypermethrin}

462 Gottardi and Cedergreen (2019) investigated the toxicity of $\alpha$-cypermethrin on Daphnia magna using an acute toxicity test following OECD guidelines for testing chemicals (Organisation for Economic Co-operation and Development (OECD), 2012b). In nine independent experiments conducted at different times, D. magma neonates ( $<1$ day old) were exposed to different concentrations of $\alpha$-cypermethrin or acetone control for 48 hours in M7 medium. Four replicates of five organisms were used for each concentration. The number of concentrations used in each sub-experiment varied from 6 to 7 (5 or 6 and control).

For this example, the purpose was to estimate BMD and BMDL for a BMR of $5 \%$ using the excess risk definition as recommended by EFSA and US EPA. However, due to the hierarchical

471 design with sub-experiments, a simple dose-response model may not appropriately capture the 472 dependence structure in data. Consequently, a two-step meta-analytic approach was applied. In 
473 the first step, a two-parameter log-logistic model was fitted to data from each sub-experiment.

474 From each model fit, the estimated BMD and the corresponding standard error were extracted. In

475 the second step, a random effects meta-analytic model was fitted using the $\mathbf{R}$ package metafor

476 (Viechtbauer, 2010).

477 Figure S3 shows the resulting model fits from step one. The estimated BMD and BMDL

478 resulting from the meta-analytic model in step 2 were $0.07 \mu \mathrm{g} / \mathrm{L}$ and $0.05 \mu \mathrm{g} / \mathrm{L}$, respectively.

\section{VALIDATION OF THE R PACKAGE}

481

482

483

484

485

486

487

488

489

490

491

492

493

A small-scale validation of the $\mathbf{R}$ package was carried out by analyzing a number of datasets using bmd and BMDS version 3.2.0 from the US. EPA and the PROAST web application ${ }^{1}$ from EFSA.

\subsection{Methods}

For binomial data, the three software packages were compared for three data sets, four different models, and two BMR levels. The three data sets used were data from experiment 4, 7, and 9 from example 3.5 corresponding to different dose-response shapes (see Figure S4). These data were analyzed using a log-logistic, a log-normal, and a Weibull model. For BMDS and bmd, two-parameter models were used, assuming lower and upper limits to be 0 and 1 , respectively. In PROAST, it was not possible to fit models with a lower limit of 0 , instead three-parameter models were fitted. BMR was set to either $5 \%$ or $10 \%$, and BMD was defined using the excess risk definition. BMDL was estimated using profile likelihood for both BMDS and PROAST, while three different alternatives were used for $b m d$ : delta method-based Wald confidence

$1 \quad$ https://shiny-efsa.openanalytics.eu/app/bmd 
494 intervals, confidence intervals obtained from inverse regression, and non-parametric bootstrap

495 confidence intervals obtained using resampling within dose groups from the original data set.

496 BMDL was found as the 5\% percentile in the bootstrap distribution.

497 For continuous data, the three software packages were compared for nine data sets, two models, 498 and two BMR levels. The nine data sets were simulated from three four-parameter log-logistic 499 models with similar lower and upper limits (2 and 10, respectively) but different location and 500 shape parameters. The three different models are shown in Figure S4. For each model, data were 501 generated at five doses $(0.1,0.5,1,5$, and 10), assuming normal distributions with i) 10 502 replicates and a standard deviation of 1, ii) 10 replicates and a standard deviation of 0.1 , or iii) 3 503 replicates and a standard deviation of 0.1 . These data were analyzed using a four-parameter log504 logistic model (called the Hill model in BMDS and PROAST) and a four-parameter Weibull 505 model (called the exponential model in BMDS and PROAST). BMR was set to either 0.05 or 506 0.1, and BMD was defined using the relative risk definition. BMDL was estimated using profile 507 likelihood for both BMDS and PROAST. For $b m d$, three alternatives for estimating BMDL were 508 applied: delta-method-based Wald confidence intervals, confidence intervals obtained from 509 inverse regression, and semi-parametric bootstrap confidence intervals obtained through 510 resampling of residuals from the dose-response model assuming exchangeability over all 511 observations. BMDL was found as the $5 \%$ percentile in the bootstrap distribution.

512 We also compared $b m d$ to BMDS and PROAST on five data sets used in the EFSA report on 513 benchmark dose modeling (Hardy et al., 2017). These data sets are described in detail below.

514 Data set 1 contains continuous data from a subchronic National Toxicity Study 416 for an 515 unknown agent. Specifically, bodyweight mean values and standard deviations are available for 
516 six dose groups of 10 animals (mg/kg body weight), including a control group. Data were

517 analyzed using a three-parameter exponential model, the relative definition, and a BMR of 5\%.

518 Data set 2 contains binomial data from a toxicity study examining the incidence of gastric

519 impaction from an unknown agent. Ten animals were used in each of four dose groups (mg/kg

520 body weight), which included a control group. Data were analyzed using a three-parameter log-

521 logistic model (with an estimated lower limit), the excess risk definition, and a BMR of $10 \%$.

522 Data set 3 comes from human dose-response study, where each individual has its exposure limit.

523 The binary endpoint is normal or abnormal eye-hand coordination in individual workers exposed

524 to different levels of CRD (unit unknown). Data were analyzed using a one-stage model, the

525 excess risk definition, and a BMR of $10 \%$.

526 Data set 4 comes from a two-year study in male mice where body weight is reported as mean

527 values, standard deviations, and sample sizes for each of four dose groups ( $\mathrm{mg} / \mathrm{kg}$ body weight)

528 of an unknown agent, including a control. Data were analyzed using a three-parameter Hill

529 model and a three-parameter exponential model using the relative definition and BMR of 5\%.

530 Finally, data set 5 contains binomial data from a two-year study in female rats examining thyroid 531 epithelial vacuolization incidence. Four dose groups ( $\mathrm{mg} / \mathrm{kg}$ body weight) of an unknown agent,

532 including a control, were considered. Data were analyzed using a three-parameter log-logistic

533 and a three-parameter log-normal model, using the excess risk definition, and a BMR of $10 \%$.

\section{$534 \quad 4.2$ Results}

535 The results for binomial and continuous data are shown in Table 2 and 3, respectively, while the

536 results for the five data sets used in the EFSA report are presented in Table 4. For the binomial

537 data, PROAST and $b m d$ agreed on BMD estimates to the third significant number for all data 
538 examples and models. BMDS agreed with $b m d$ for most of the models, except the Weibull model

539 in a few cases. For continuous data, bmd and BMDS agreed on the BMD estimate to the third

540 significant number with only a few exceptions. PROAST gave quite different results for most

541 scenarios.

542 BMDL estimates from $b m d$ did not match those from BMDS and PROAST as these were based

543 on different methods. However, for binomial data the delta method derived BMDL estimates

544 from $b m d$ were generally close to the BMDL estimates from both PROAST and BMDS. For

545 continuous data, the bootstrap BMDL estimates from $b m d$ were generally very close to BMDL

546 estimated by BMDS. From the results presented here, it is not possible to say which of the

547 different approaches performs best.

548 The running time for some of the functionality involving bootstrap may be rather long. However,

549 it is mainly an issue for simulation purposes where hundreds or thousands of estimates are to be

550 found. In addition, comparisons to PROAST web-application shows shorter run times for $b m d$.

\section{5. DISCUSSION}

552 We have described in detail the functionality of the $\mathbf{R}$ package $b m d$ for BMD estimation. The

553 usefulness and flexibility of tbmd were illustrated by means of a number of examples, covering

554 both simple and more complex data structures. In the first example, binomial data for a single

555 dose-response curve was used to estimate BMD and BMDL. In the second and third example, we

556 revisited a data set with count data and considered different ways of approaching the related

557 challenges, including potential overdispersion of the counts. The fourth data example illustrated

558 the flexibility of the package to estimate a model-averaged BMD and BMDL, whereas the last

559 example showed how to handle data from more complex experimental designs. 
560 The package $b m d$ differs from the existing specialized BMD software packages BMDS and

561 PROAST as it provides BMD estimation for several types of response data, including count data

562 and time-to-event data, two types of data that often occur in ecotoxicology. Count data are often

563 handled by assuming a normal or log-normal distribution ignoring that such data cannot be

564 negative, may present ties and usually experience variances that are a function of the mean and

565 accordingly violates the assumption of variance homogeneity, i.e., for the special case of Poisson

566 distributed variables, the variance equals the mean. Though transformations may accommodate

567 some of the issues related to count data, it has been shown that they result in a lack of power

568 compared to statistically sound alternatives, such as using the Poisson or negative binomial

569 distribution (Stroup, 2013, 2015).

570 The package $b m d$ provides BMD estimation for practically all dose-response functions available 571 in BMDS and PROAST. The package also allows the use of fractional polynomials, particularly

572 suitable for model averaging (Faes et al., 2007; Namata et al., 2008), and several hormesis

573 models, as well as semi-parametric alternatives (Piegorsch et al., 2014; Lin, Piegorsch \&

574 Bhattacharya, 2015). An additional advantage of $b m d$ is the possibility to estimate BMDL based

575 on sandwich variance-covariance estimates that provide robust standard errors, thus making

576 allowance for model misspecification related to the distributional assumptions. This option is not

577 available in any other specialized BMD software packages. Several different types of bootstrap

578 can be used to estimate BMDL, and several options for estimating BMD and BMDL, including

579 model averaging, are available. Also, other available or future software extensions of $\mathbf{R}$ can be

580 directly used together with $b m d$.

581 When comparing results from $b m d$, BMDS, and PROAST, we mostly observed agreement

582 between estimated BMD and BMDL values. However, we also found some differences. We 
583 found small differences that most likely were caused by different underlying optimization

584 algorithms. In some scenarios, we also found larger differences. Differences between

$585 \mathrm{bmd} / \mathrm{BMDS}$ on one side and PROAST on the other side were most likely due to the fact that

586 PROAST assumed a log-normal distribution whereas BMDS/bmd assumed normal distributions.

587 Depending on the scale for the response, these two assumptions may lead to fairly different

588 results. Moreover, there could also easily be differences due to the different methods used for

589 obtaining confidence intervals and hence BMDL estimates.

590 At present, bmd can only handle models describing a single dose-response curve. However, a

591 future update will extend the built-in functions to handle models for multiple dose-response

592 curves. It would be interesting to include an approach for BMDL estimation for model-averaged

593 BMD where correlations between BMD estimates from the different candidate models would be

594 accounted for (Jensen \& Ritz, 2015). Likewise, it would be interesting to look at other

595 approaches or models for addressing non-constant variance than assuming log-normally

596 distributed data. Finally, the profile likelihood approach for finding BMDL has been found to

597 perform better than the delta-method-based Wald confidence intervals (Moerbeek, Piersma \&

598 Slob, 2004; US Environmental Protection Agency, 2012). This approach is currently not

599 available in $b m d$ but is planned to be part of a future update.

600 Subsection 2.1 illustrates that the BMD directly depends on its formulation, which needs to be

601 clearly defined and may thus be perceived as superficially more technically involved than

602 statistical testing. Thus, the BMD approach may be considered a statistical black box, which may

603 affect its use in practice. However, it is accepted or even preferred to the NOAEL approach due

604 to its benefits, as laid out in the introduction. Further, statistical testing itself is scrutinized when

605 used as a naïve binary decision criterion (e.g., Wasserstein et al., 2019; Wasserstein \& Lazar, 
606 2016) and with respect to toxicology (Kluxen \& Hothorn, 2020; Hothorn \& Pirow, 2020;

607 Kluxen, 2020).

608

\section{CONCLUSION}

610 We have demonstrated that the $\mathbf{R}$ extension package $b m d$ allows flexible and straightforward 611 BMD estimation for a broad range of applications in ecotoxicology. Comparisons to existing 612 software showed mostly good agreement between estimates, but also some non-negligible 613 differences due to different statistical methods being used. However, we observed differences 614 between all three software programs: $b m d$, BMDS, and PROAST. The freely available $\mathbf{R}$ 615 software can be viewed as a reliable and user-friendly alternative for dose-response modelling, 616 which also covers all other conceivable practical statistical requirements for (eco)toxicologists, 617 including BMD analyses.

\section{ACKNOWLEDGEMENTS}

621 We would like to thank the three reviewers for their helpful and detailed comments and many 622 practical suggestions.

\section{REFERENCES}

625 Bailer AJ, Noble RB, Wheeler MW. 2005. Model Uncertainty and Risk Estimation for 626 Experimental Studies of Quantal Responses. Risk Analysis 25:291-299. DOI: 10.1111/j.1539-6924.2005.00590.x. 
628 Brescia S. 2020. Thresholds of adversity and their applicability to endocrine disrupting

629 chemicals. Critical Reviews in Toxicology 50:213-218. DOI:

$630 \quad 10.1080 / 10408444.2020 .1740973$.

631 Buckland ST, Burnham KP, Augustin NH. 1997. Model Selection: An Integral Part of Inference. 632 Biometrics 53:603-618. DOI: 10.2307/2533961.

633 Buckley BE, Piegorsch WW, West RW. 2009. Confidence limits on one-stage model parameters 634 in benchmark risk assessment. Environmental and ecological statistics 16:53-62. DOI: $635 \quad 10.1007 / \mathrm{s} 10651-007-0076-2$.

636 Budtz-Jørgensen E, Keiding N, Grandjean P. 2001. Benchmark Dose Calculation from 637 Epidemiological Data. Biometrics 57:698-706. DOI: 10.1111/j.0006-341X.2001.00698.x.

638 Carroll RJ, Ruppert D. 1988. Transformation and weighting in regression. London: Chapman \& 639 Hall.

640 Cedergreen N, Nørhave NJ, Svendsen C, Spurgeon DJ. 2016. Variable Temperature Stress in the 641 Nematode Caenorhabditis elegans (Maupas) and Its Implications for Sensitivity to an 642 Additional Chemical Stressor. PLOS ONE 11:e0140277. DOI:

$643 \quad$ 10.1371/journal.pone.0140277.

644 Cedergreen N, Streibig JC, Spliid NH. 2004. Sensitivity of aquatic plants to the herbicide 645 metsulfuron-methyl. Ecotoxicology and Environmental Safety 57:153-161. DOI:

$646 \quad 10.1016 / \mathrm{S} 0147-6513(02) 00145-8$.

647 Claeskens G, Hjort NL. 2008. Model selection and model averaging. Cambridge: Cambridge 648 University Press. 
649 Crump KS. 1984. A new method for determining allowable daily intakes. Fundamental and 650 Applied Toxicology 4:854-871. DOI: 10.1016/0272-0590(84)90107-6.

651 Crump KS. 1995. Calculation of Benchmark Doses from Continuous Data. Risk Analysis 15:79652 89. DOI: $10.1111 /$ j.1539-6924.1995.tb00095.x.

653 Davis JA, Gift JS, Zhao QJ. 2011. Introduction to benchmark dose methods and US EPA's 654 benchmark dose software (BMDS) version 2.1.1. Toxicology and Applied Pharmacology 655 254:181-191. DOI: 10.1016/J.TAAP.2010.10.016.

656

657

658

659

660

661

662

663

664 665

666

667

668

669

Delignette-Muller ML, Lopes C, Veber P, Charles S. 2014. Statistical Handling of Reproduction Data for Exposure-Response Modeling. Environmental Science and Technology 48:75447551. DOI: 10.1021/es502009r.

Faes C, Aerts M, Geys H, Molenberghs G. 2007. Model Averaging Using Fractional Polynomials to Estimate a Safe Level of Exposure. Risk Analysis 27:111-123. DOI: 10.1111/j.1539-6924.2006.00863.x.

Fang Q, Piegorsch WW, Barnes KY. 2015. Bayesian benchmark dose analysis. Environmetrics 26:373-382. DOI: 10.1002/env.2339.

Gaylor DW, Slikker W. 1990. Risk assessment for neurotoxic effects. Neurotoxicology 11:2118.

Gottardi M, Cedergreen N. 2019. The synergistic potential of azole fungicides does not directly correlate to the inhibition of cytochrome P450 activity in aquatic invertebrates. Aquatic Toxicology 207:187-196. DOI: 10.1016/J.AQUATOX.2018.12.010.

Haber LT, Dourson ML, Allen BC, Hertzberg RC, Parker A, Vincent MJ, Maier A, Boobis AR. 
670

671

672

673

674

675

676

677

678

679

680

681

682

683

684

685

686

687

688

689

690

2018. Benchmark dose (BMD) modeling: current practice, issues, and challenges. Critical Reviews in Toxicology 48:387-415. DOI: 10.1080/10408444.2018.1430121.

Hardy A, Benford D, Halldorsson T, Jeger MJ, Knutsen KH, More S, Mortensen A, Naegeli H, Noteborn H, Ockleford C, Ricci A, Rychen G, Silano V, Solecki R, Turck D, Aerts M, Bodin L, Davis A, Edler L, Gundert-Remy U, Sand S, Slob W, Bottex B, Abrahantes JC, Marques DC, Kass G, Schlatter JR. 2017. Update: use of the benchmark dose approach in risk assessment. EFSA Journal 15:1-41. DOI: 10.2903/j.efsa.2017.4658.

Hoekstra JA. 1987. Acute bioassays with control mortality. Water, Air, and Soil Pollution 35:311-317. DOI: 10.1007/BF00290938.

Hothorn LA, Ralph Pirow. 2020. Use compatibility intervals in regulatory toxicology. Regulatory Toxicology and Pharmacology 116:104720. DOI: 10.1016/j.yrtph.2020.104720.

Jensen SM, Kluxen FM, Ritz C. 2019. A Review of Recent Advances in Benchmark Dose Methodology. Risk Analysis 39:2295-2315. DOI: 10.1111/risa.13324.

Jensen SM, Ritz C. 2015. Simultaneous Inference for Model Averaging of Derived Parameters. Risk Analysis 35:68-76. DOI: 10.1111/risa.12242.

Jiang X, Kopp-Schneider A. 2014. Summarizing EC50 estimates from multiple dose-response experiments: A comparison of a meta-analysis strategy to a mixed-effects model approach. Biometrical Journal 56:493-512. DOI: 10.1002/bimj.201300123.

Kang S-H, Kodell RL, Chen JJ. 2000. Incorporating Model Uncertainties along with Data Uncertainties in Microbial Risk Assessment. Regulatory Toxicology and Pharmacology 32:68-72. DOI: 10.1006/RTPH.2000.1404. 
691 Kennedy-Shaffer L. 2019. Before $p$ \&lt; 0.05 to Beyond $p$ \&lt; 0.05: Using History to

692 Contextualize $p$-Values and Significance Testing. The American Statistician 73:82-90.

693 DOI: $10.1080 / 00031305.2018 .1537891$.

694 Kluxen FM. 2020. "New statistics" in regulatory toxicology? Regulatory Toxicology and

695 Pharmacology 117:104763. DOI: 10.1016/j.yrtph.2020.104763.

696 Kluxen FM, Hothorn LA. 2020. Alternatives to statistical decision trees in regulatory (eco-

697 )toxicological bioassays. Archives of Toxicology 94:1135-1149. DOI: 10.1007/s00204-020-

$698 \quad$ 02690-w.

699 Lin L, Piegorsch WW, Bhattacharya R. 2015. Nonparametric Benchmark Dose Estimation with

700 Continuous Dose-Response Data. Scandinavian Journal of Statistics 42:713-731. DOI:

$701 \quad 10.1111 /$ sjos.12132.

702 Moerbeek M, Piersma AH, Slob W. 2004. A Comparison of Three Methods for Calculating

703 Confidence Intervals for the Benchmark Dose. Risk Analysis 24:31-40. DOI:

$704 \quad 10.1111 / \mathrm{j} .0272-4332.2004 .00409 . x$.

705 Moon H, Kim H-J, Chen JJ, Kodell RL. 2005. Model Averaging Using the Kullback Information

706 Criterion in Estimating Effective Doses for Microbial Infection and Illness. Risk Analysis

707 25:1147-1159. DOI: $10.1111 / \mathrm{j} .1539-6924.2005 .00676 . x$.

708 Namata H, Aerts M, Faes C, Teunis P. 2008. Model Averaging in Microbial Risk Assessment

$709 \quad$ Using Fractional Polynomials. Risk Analysis 28:891-905. DOI: 10.1111/j.1539-

$710 \quad 6924.2008 .01063 . x$

711 Organisation for Economic Co-operation and Development (OECD). 2006. Current approaches 
in the statistical analysis of ecotoxicity data: A guidance to application - Annexes.

713 Organisation for Economic Co-operation and Development (OECD). 2012a. Guidance document

714116 on the conduct and design of chronic toxicity and carcinogenicity studies, supporting

715 test guidelines 451, 452 and 453. Paris.

716 Organisation for Economic Co-operation and Development (OECD). 2012b. Test No. 211:

717 Daphnia magna Reproduction Test. OECD. DOI: 10.1787/9789264185203-en.

718 Piegorsch WW. 2010. Translational benchmark risk analysis. Journal of Risk Research 13:653-

719 667. DOI: $10.1080 / 13669870903551662$.

Piegorsch WW, Xiong H, Bhattacharya RN, Lin L. 2012. Non-parametric estimation of benchmark doses in environmental risk assessment. Environmetrics 23:717-728. DOI:

Piegorsch WW, Xiong H, Bhattacharya RN, Lin L. 2014. Benchmark Dose Analysis via Nonparametric Regression Modeling. Risk Analysis 34:135-151. DOI: 10.1111/risa.12066.

R Core Team. 2018. R: A language and environment for statistical computing.

726

727

728

729

730

731

732 10.1002/env.2175.

Ringblom J, Johanson G, Öberg M. 2014. Current modeling practice may lead to falsely high benchmark dose estimates. Regulatory Toxicology and Pharmacology 69:171-177. DOI: 10.1016/J.YRTPH.2014.03.004.

Ritz C, Baty F, Streibig JC, Gerhard D. 2015. Dose-response analysis using R. PloS one 10:e0146021. DOI: 10.1371/journal.pone.0146021.

Ritz C, Gerhard D, Hothorn LA. 2013. A Unified Framework for Benchmark Dose Estimation Applied to Mixed Models and Model Averaging. Statistics in Biopharmaceutical Research 
734

735

736

737

Ritz C, Jensen SM, Gerhard D, Streibig JC. 2019. Dose-response analysis using R. Boca Raton, Florida: Chapman \& Hall.

Salsburg D. 2001. The lady tasting tea: How statistics revolutionized science in the twentieth century. Macmillan.

Shao K, Shapiro AJ. 2018. A Web-Based System for Bayesian Benchmark Dose Estimation. Environmental Health Perspectives 126:017002. DOI: 10.1289/EHP1289.

Slob W. 2002. Dose-Response Modeling of Continuous Endpoints. Toxicological Sciences 66:298-312. DOI: 10.1093/toxsci/66.2.298.

Slob W. 2017. A general theory of effect size, and its consequences for defining the benchmark response (BMR) for continuous endpoints. Critical Reviews in Toxicology 47:342-351. DOI: $10.1080 / 10408444.2016 .1241756$.

Slob W. 2018. Joint project on Benchmark Dose modelling with RIVM. EFSA Supporting Publications 15:1-14. DOI: 10.2903/sp.efsa.2018.EN-1497.

Stroup WW. 2013. Non-normal data in agricultural experiments. In: Conference on Applied Statistics in Agriculture. New Prairie Press, 103-135. DOI: 10.4148/2475-7772.1018.

Stroup WW. 2015. Rethinking the Analysis of Non-Normal Data in Plant and Soil Science. Agronomy Journal 107:811. DOI: 10.2134/agronj2013.0342.

US Environmental Protection Agency. 2012. Benchmark Dose Technical Guidance. Washington, DC.

Varewyck M, Verbeke T. 2017. Software for benchmark dose modelling. EFSA Supporting 
755 Viechtbauer W. 2010. Conducting Meta-Analyses in R with the metafor package. Journal of $756 \quad$ Statistical Software 36:1-48.

757 Wang M, Riffel M. 2011. Making the right conclusions based on wrong results and small sample 758 sizes: Interpretation of statistical tests in ecotoxicology. Ecotoxicology and Environmental 759 Safety 74:684-692. DOI: 10.1016/j.ecoenv.2010.10.019.

760 Wasserstein RL, Lazar NA. 2016. The ASA's Statement on p-Values: Context, Process, and Purpose. American Statistician 70:129-133. DOI: 10.1080/00031305.2016.1154108.

762

763

764

765

766

767

768

769

770

771

772

773

774

Wasserstein RL, Schirm AL, Lazar NA. 2019. Moving to a World Beyond " $p<0.05$." American Statistician 73:1-19. DOI: 10.1080/00031305.2019.1583913.

West RW, Piegorsch WW, Peña EA, An L, Wu W, Wickens AA, Xiong H, Chen W. 2012. The impact of model uncertainty on benchmark dose estimation. Environmetrics 23:706-716. DOI: $10.1002 /$ env.2180.

Wheeler MW, Bailer AJ. 2007. Properties of Model-Averaged BMDLs: A Study of Model Averaging in Dichotomous Response Risk Estimation. Risk Analysis 27:659-670. DOI: 10.1111/j.1539-6924.2007.00920.x.

Wheeler MW, Bailer AJ. 2008. Model Averaging Software for Dichotomous Dose Response Risk Estimation. Journal of Statistical Software 26:1-15. DOI: 10.18637/jss.v026.i05.

Wheeler MW, Bailer AJ. 2009. Benchmark Dose Estimation Incorporating Multiple Data Sources. Risk Analysis 29:249-256. DOI: 10.1111/j.1539-6924.2008.01144.x.

Wu Y, Piegorsch WW, West RW, Tang D, Petkewich MO, Pan W. 2006. Multiplicity-adjusted 
775 Inferences in Risk Assessment: Benchmark Analysis with Continuous Response Data. Environmental and Ecological Statistics 13:125-141. DOI: 10.1007/s10651-005-5695-x.

777 Yang L, Allen BC, Thomas RS. 2007. BMDExpress: a software tool for the benchmark dose 778 analyses of genomic data. BMC Genomics 8:387. DOI: 10.1186/1471-2164-8-387. 
Figure 1

The fitted concentration-response curve for the three-parameter log-normal model fitted to data from the earthworm toxicity test, with different concentrations of chloroacetamide (mg/kg soil) (Hoekstra, 1987).

The model is shown together with data (mean values per dose)concentration), and the estimated benchmark dose (BMD) and benchmark dose lower limit (BMDL for) based on the added risk definition. and a benchmark response (BMR) of $0.05 . \mathrm{p} 0$ is the estimated probability of dying for the background population, i.e. the background response level.

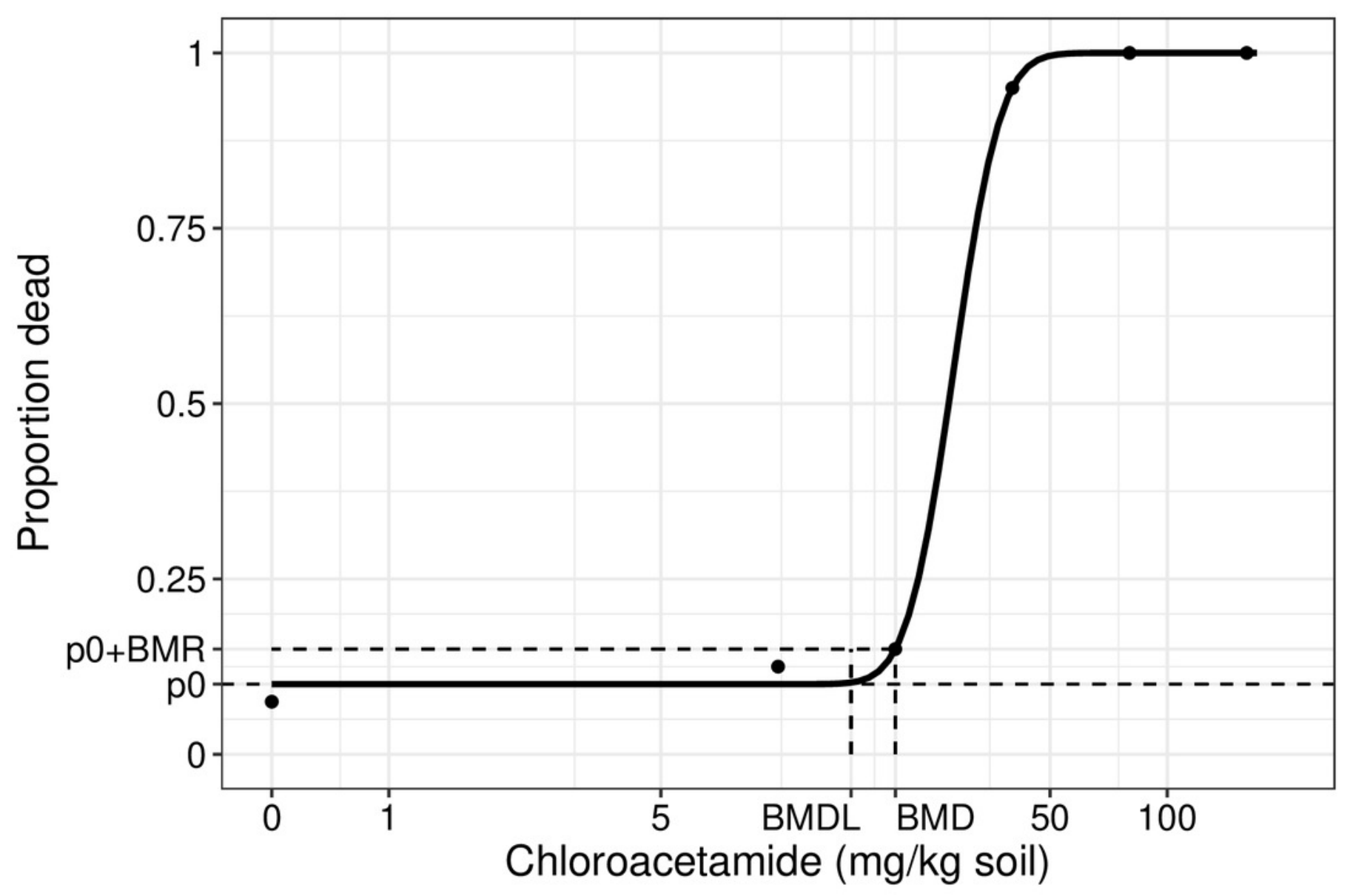


Figure 2

Fitted concentration-response curve for the two-parameter exponential decay model, the three-parameter log-logistic model and the two different Weibull models fitted to the Rainbow trout data, (Organisation for Economic Co-operation and Development (OECD)

The curve is shown together with all data points. The Rainbow trouts were exposed to different concentrations (mg/L water) of an unknown agent.

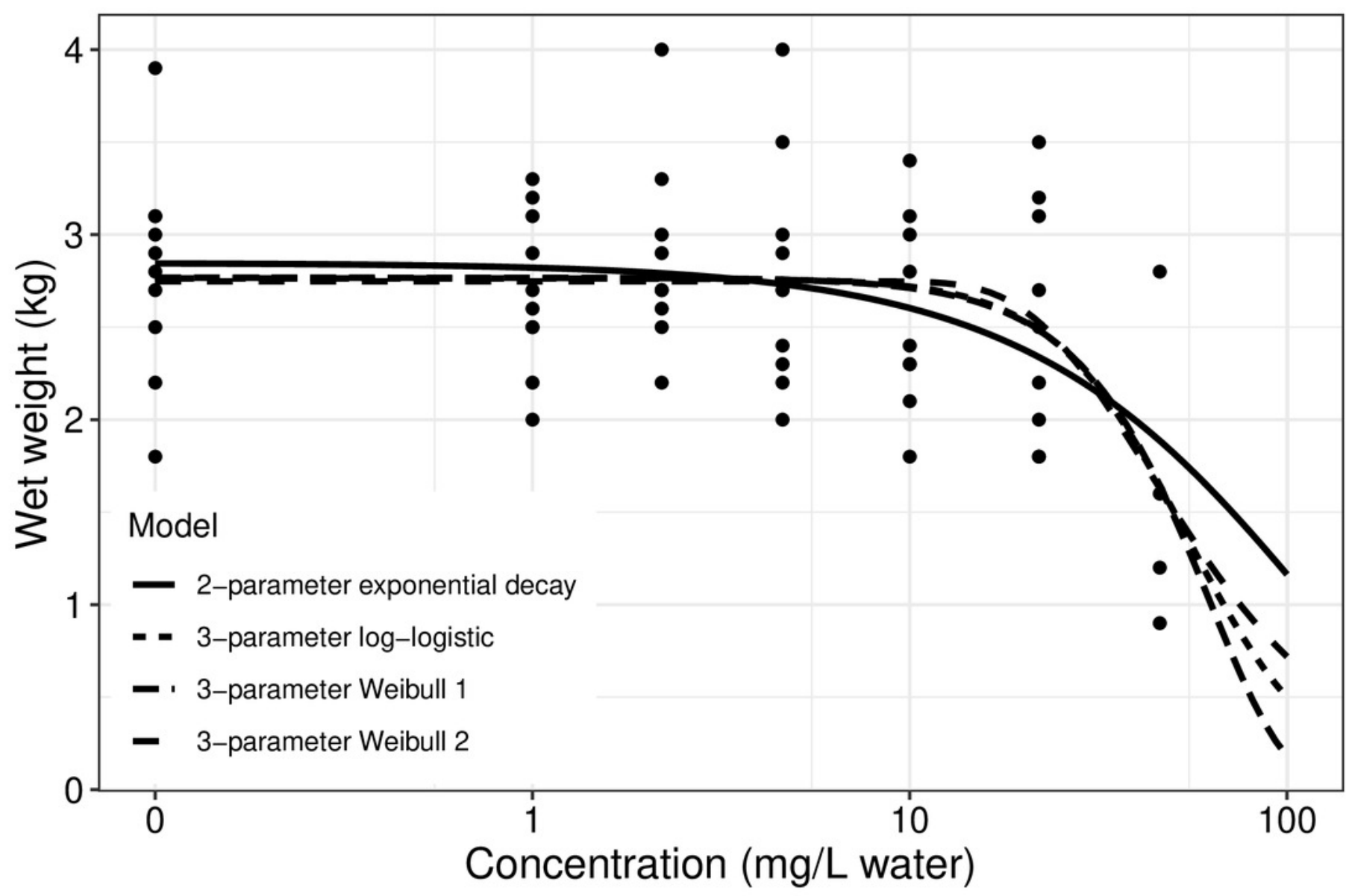




\section{Table $\mathbf{1}$ (on next page)}

Resulting benchmark dose (BMD), benchmark dose lower limit (BMDL), Akaike information criteria (AIC), and weight based on AIC for four different models fitted to data from a fish test with Rainbow trout.

BMD was estimated based on the hybrid approach using 2 standard deviations as the cutoff and $B M R=0.5$. The model-averaged BMDLs were based on non-parametric bootstrap. 
Table 1: Resulting benchmark dose (BMD), benchmark dose lower limit (BMDL), Akaike information criteria (AIC), and weight based on AIC for four different models fitted to data from a fish test with Rainbow trout. BMD was estimated based on the hybrid approach using 2 standard deviations as the cutoff and $B M R=0.5$. The model-averaged BMDLs were based on non-parametric bootstrap.

\begin{tabular}{|c|c|c|c|c|c|}
\hline & Parameters & $\begin{array}{l}\text { BMD } \\
(\mathrm{mg} / \mathrm{L})\end{array}$ & $\begin{array}{l}\text { BMDL } \\
(\mathrm{mg} / \mathrm{L})\end{array}$ & AIC & Weight \\
\hline Exponential decay & 2 & 12.65 & 6.37 & 106.31 & 0.333 \\
\hline Log-logistic & 3 & 22.91 & 9.05 & 106.65 & 0.237 \\
\hline Weibull type 1 & 3 & 22.72 & 8.12 & 106.58 & 0.253 \\
\hline Weibull type 2 & 3 & 24.26 & 14.26 & 106.94 & 0.177 \\
\hline $\begin{array}{l}\text { Model averaging } \\
\text { (estimate averaging) }\end{array}$ & & 19.68 & 8.04 & & \\
\hline $\begin{array}{l}\text { Model averaging } \\
\text { (curve averaging) }\end{array}$ & & 20.35 & 7.73 & & \\
\hline
\end{tabular}




\section{Table 2 (on next page)}

Estimated benchmark dose (BMD) and benchmark dose lower limit (BMDL) for different binomial data sets, different models and different levels of BMR using PROAST, BMDS and bmd.

PROAST and BMDS uses profile likelihood intervals for estimating BMDL while the R package bmd uses the delta method, inverse regression or bootstrap. For all data sets the excess risk definition was used. 
1 Table 2: Estimated benchmark dose (BMD) and benchmark dose lower limit (BMDL) for

2 different binomial data sets, different models and different levels of BMR using PROAST, BMDS

3 and $\mathrm{bmd}$. PROAST and BMDS uses profile likelihood intervals for estimating BMDL while the $\boldsymbol{R}$

4 package bmd uses the delta method, inverse regression or bootstrap. For all data sets the excess

5 risk definition was used.

\begin{tabular}{|c|c|c|c|c|c|c|c|c|c|c|}
\hline \multirow[b]{3}{*}{ Data } & \multirow[b]{3}{*}{ Model $^{1}$} & \multirow[b]{3}{*}{ BMR } & \multicolumn{3}{|c|}{ BMD } & \multicolumn{5}{|c|}{ BMDL } \\
\hline & & & & & & PROAST & BMDS & bmd & bmd & bmd \\
\hline & & & PROAST & BMDS & bmd & profile & profile & delta & inverse & bootstrap \\
\hline \multirow[t]{6}{*}{ A } & Log-logistic & 0.05 & 0.044 & 0.044 & 0.044 & 0.022 & 0.022 & 0.020 & 0.029 & 0.027 \\
\hline & & 0.1 & 0.063 & 0.062 & 0.062 & 0.036 & 0.036 & 0.036 & 0.043 & 0.042 \\
\hline & Log-normal & 0.05 & 0.047 & 0.047 & 0.047 & 0.026 & 0.026 & 0.025 & 0.033 & 0.030 \\
\hline & & 0.1 & 0.063 & 0.063 & 0.063 & 0.038 & 0.038 & 0.038 & 0.045 & 0.043 \\
\hline & Weibull & 0.05 & 0.027 & 0.027 & 0.027 & 0.010 & 0.010 & 0.006 & 0.014 & 0.013 \\
\hline & & 0.1 & 0.047 & 0.047 & 0.047 & 0.022 & 0.022 & 0.018 & 0.027 & 0.026 \\
\hline \multirow[t]{6}{*}{ B } & Log-logistic & 0.05 & 0.087 & 0.087 & 0.087 & 0.063 & 0.063 & 0.064 & 0.071 & 0.072 \\
\hline & & 0.1 & 0.102 & 0.102 & 0.102 & 0.079 & 0.079 & 0.080 & 0.085 & 0.086 \\
\hline & Log-normal & 0.05 & 0.089 & 0.089 & 0.089 & 0.066 & 0.066 & 0.066 & 0.074 & 0.075 \\
\hline & & 0.1 & 0.102 & 0.102 & 0.102 & 0.079 & 0.079 & 0.080 & 0.085 & 0.087 \\
\hline & Weibull & 0.05 & 0.078 & 0.072 & 0.078 & 0.049 & 0.049 & 0.049 & 0.058 & 0.059 \\
\hline & & 0.1 & 0.098 & 0.093 & 0.098 & 0.069 & 0.068 & 0.069 & 0.076 & 0.078 \\
\hline \multirow[t]{3}{*}{$\mathrm{C}$} & Log-logistic & 0.05 & 0.055 & 0.55 & 0.055 & 0.032 & 0.031 & 0.029 & 0.037 & 0.035 \\
\hline & & 0.1 & 0.081 & 0.081 & 0.081 & 0.052 & 0.052 & 0.050 & 0.058 & 0.056 \\
\hline & Log-normal & 0.05 & 0.058 & 0.058 & 0.058 & 0.036 & 0.036 & 0.034 & 0.041 & 0.039 \\
\hline
\end{tabular}




\begin{tabular}{cccccccccccc} 
& 0.1 & 0.080 & 0.080 & 0.080 & 0.053 & 0.053 & 0.052 & 0.058 & 0.057 \\
Weibull & 0.05 & 0.046 & 0.046 & 0.046 & 0.024 & 0.024 & 0.020 & 0.029 & 0.028 \\
& 0.1 & 0.077 & 0.077 & 0.077 & 0.046 & 0.046 & 0.043 & 0.051 & 0.051 \\
\hline
\end{tabular}

${ }^{1}$ All models fitted as unrestricted models in BMDS. 


\section{Table 3 (on next page)}

Estimated benchmark dose (BMD) and benchmark dose lower limit (BMDL) for different continuous data sets, different models and different levels of BMR using PROAST, BMDS and bmd.

PROAST and BMDS uses profile likelihood intervals for estimating BMDL while the R package bmd uses the delta method, inverse regression or bootstrap. For all data sets, the relative definition of BMD was used. 
Table 3: Estimated benchmark dose (BMD) and benchmark dose lower limit (BMDL) for different continuous data sets, different

models and different levels of BMR using PROAST, BMDS and bmd. PROAST and BMDS uses profile likelihood intervals for

definition of BMD was used.

\section{BMD}

Data

set Std Rep Model

\begin{tabular}{|c|c|c|c|c|c|c|c|c|c|c|c|c|}
\hline \multirow[b]{2}{*}{ Dat } & \multirow[b]{3}{*}{ Std } & \multirow[b]{3}{*}{ Rep } & \multirow[b]{3}{*}{ Model } & \multirow[b]{3}{*}{ BMR } & \multirow[b]{3}{*}{ PROAST } & \multirow[b]{3}{*}{ BMDS } & \multirow[b]{3}{*}{ bmd } & & & \multirow{3}{*}{$\begin{array}{l}\text { bmd } \\
\text { delta }\end{array}$} & \multirow{3}{*}{$\begin{array}{l}\text { bmd } \\
\text { inverse }\end{array}$} & \multirow{3}{*}{$\begin{array}{l}\text { bmd } \\
\text { bootstrap }\end{array}$} \\
\hline & & & & & & & & PROAST & BMDS & & & \\
\hline set & & & & & & & & profile & profile & & & \\
\hline \multirow[t]{9}{*}{$\mathrm{A}$} & 1 & 10 & Log-logistic $^{1}$ & 0.05 & 0.088 & 0.144 & 0.144 & 0 & 0.050 & 0.037 & 0.082 & 0.051 \\
\hline & & & & 0.1 & 0.129 & 0.200 & 0.200 & 0 & 0.080 & 0.077 & 0.119 & 0.080 \\
\hline & & & Weibull $^{2}$ & 0.05 & 0.040 & 0.126 & 0.126 & 0 & 0.018 & 0.004 & 0.063 & 0.017 \\
\hline & & & & 0.1 & 0.070 & 0.183 & 0.183 & 0 & 0.034 & 0.038 & 0.097 & 0.032 \\
\hline & 0.1 & 10 & Log-logistic & 0.05 & 0.101 & 0.107 & 0.107 & 0.091 & 0.105 & 0.097 & 0.097 & 0.096 \\
\hline & & & & 0.1 & 0.148 & 0.154 & 0.154 & 0.135 & 0.151 & 0.142 & 0.140 & 0.140 \\
\hline & & & Weibull & 0.05 & 0.049 & 0.074 & 0.074 & 0.041 & 0.060 & 0.061 & 0.061 & 0.061 \\
\hline & & & & 0.1 & 0.086 & 0.115 & 0.115 & 0.073 & 0.097 & 0.099 & 0.098 & 0.098 \\
\hline & 0.1 & 3 & Log-logistic & 0.05 & 0.092 & 0.097 & 0.097 & 0.080 & 0.078 & 0.075 & 0.077 & 0.079 \\
\hline
\end{tabular}

\section{BMDL}




\begin{tabular}{|c|c|c|c|c|c|c|c|c|c|c|c|c|}
\hline & & & & 0.1 & 0.138 & 0.140 & 0.140 & 0.121 & 0.1156 & 0.114 & 0.114 & 0.117 \\
\hline & & & Weibull & 0.05 & 0.043 & 0.066 & 0.066 & 0.031 & 0.040 & 0.037 & 0.043 & 0.043 \\
\hline \multirow{3}{*}{\multicolumn{2}{|c|}{ B }} & & & 0.1 & 0.008 & 0.148 & 0.149 & 0 & 0.011 & 0 & 0.06 & 0.012 \\
\hline & & & Weibull & 0.05 & 0 & 0.045 & 0.045 & 0 & 0.015 & 0 & 0.014 & 0.003 \\
\hline & & & & 0.1 & 0.002 & 0.085 & 0.085 & 0 & 0.030 & 0 & 0.029 & 0.007 \\
\hline \multirow{2}{*}{\multicolumn{2}{|c|}{0.1}} & & Weibull & 0.05 & 0.008 & 0.056 & 0.016 & 0.003 & 0.051 & 0.011 & 0.011 & 0.011 \\
\hline & & & & 0.1 & 0.022 & 0.112 & 0.038 & 0.012 & 0.102 & 0.029 & 0.027 & 0.027 \\
\hline & 0.1 & 3 & Log-logistic & 0.05 & 0.029 & 0.036 & 0.036 & 0.016 & 0.028 & 0.024 & 0.023 & 0.023 \\
\hline & & & & 0.1 & 0.064 & 0.074 & 0.074 & 0.038 & 0.051 & 0.055 & 0.051 & 0.051 \\
\hline & & & Weibull & 0.05 & 0.008 & 0.056 & 0.019 & 0.004 & 0.049 & 0.012 & 0.012 & 0.012 \\
\hline & & & & 0.1 & 0.023 & 0.113 & 0.044 & 0.012 & 0.099 & 0.031 & 0.029 & 0.03 \\
\hline
\end{tabular}




\begin{tabular}{|c|c|c|c|c|c|c|c|c|c|c|}
\hline 110 & Log-logistic ${ }^{3}$ & 0.05 & - & 0.717 & 0.717 & - & 0.047 & 0 & 0.261 & 0.079 \\
\hline \multirow{3}{*}{1} & & 0.1 & - & 0.971 & 0.971 & - & 0.098102 & 0 & 0.369 & 0.155 \\
\hline & Weibull & 0.05 & - & 0.666 & 0.666 & - & 0.051 & 0 & 0.224 & 0.067 \\
\hline & & 0.1 & - & 0.930 & 0.930 & - & 0.102 & 0 & 0.329 & 0.134 \\
\hline \multirow[t]{4}{*}{0.1} & Log-logistic & 0.05 & 0.847 & 0.843 & 0.843 & 0.737 & 0.832 & 0.683 & 0.696 & 0.693 \\
\hline & & 0.1 & 1.137 & 1.126 & 1.126 & 1.010 & 1.111 & 0.954 & 0.953 & 0.957 \\
\hline & Weibull & 0.05 & 0.827 & 0.833 & 0.833 & 0.705 & 0.677 & 0.651 & 0.679 & 0.677 \\
\hline & & 0.1 & 1.155 & 1.132 & 1.132 & 1.010 & 0.950 & 0.929 & 0.948 & 0.952 \\
\hline \multirow[t]{4}{*}{0.13} & Log-logistic & 0.05 & 1.724 & 3.262 & 2.621 & 1.070 & 3.219 & 0 & 1.501 & 0.821 \\
\hline & & 0.1 & 2.054 & 3.491 & 2.905 & 1.380 & 3.459 & 0 & 1.684 & 1.107 \\
\hline & Weibull & 0.05 & 2.007 & 3.533 & 2.647 & 1.090 & 3.431 & 0 & 1.513 & 0.802 \\
\hline & & 0.1 & 2.382 & 3.753 & 2.956 & 1.450 & 3.329 & 0 & 1.716 & 1.095 \\
\hline
\end{tabular}

\footnotetext{
${ }^{1}$ Hill model for BMDS and PROAST. The Hill model was fitted as an unrestricted model in BMDS.

${ }^{2}$ Exponential model in BMDS and PROAST. The exponential model was fitted as a restricted model in BMDS.

${ }^{3}$ No model fitted for this data set using PROAST
} 


\section{Table 4 (on next page)}

Estimated benchmark dose (BMD) and benchmark dose lower limit (BMDL) for five data sets used in the Benchmark dose report from EFSA (Hardy et al., 2017).

Data were analyzed using PROAST, BMDS and bmd. PROAST and BMDS uses profile likelihood intervals for estimating BMDL while the R package bmd uses the delta method, inverse regression or bootstrap. 
Table 4: Estimated benchmark dose (BMD) and benchmark dose lower limit (BMDL) for five data sets used in the Benchmark dose

2 report from EFSA(Hardy et al., 2017). Data were analyzed using PROAST, BMDS and bmd. PROAST and BMDS uses profile

3 likelihood intervals for estimating BMDL while the $\boldsymbol{R}$ package bmd uses the delta method, inverse regression or bootstrap.

\begin{tabular}{|c|c|c|c|c|c|c|c|c|c|c|c|c|}
\hline \multirow[b]{3}{*}{ Data } & \multirow[b]{3}{*}{ Data type } & \multirow[b]{3}{*}{ Definition } & \multirow[b]{3}{*}{ BMR } & \multirow[b]{3}{*}{ Model } & \multicolumn{3}{|l|}{ BMD } & \multicolumn{4}{|c|}{ BMDL } & \multirow[b]{2}{*}{ bmd } \\
\hline & & & & & & & & PROAST & BMDS & bmd & bmd & \\
\hline & & & & & PROAST & BMDS & bmd & profile & profile & delta & inverse & bootstrap \\
\hline 1 & Continuous & Relative & $5 \%$ & Exponential & 235.1 & 233.7 & 235.5 & 170 & 170.4 & 201.5 & 203.3 & 201.1 \\
\hline 2 & Binomial & Excess & $10 \%$ & Log-logistic & 399 & 398.6 & 398.7 & 171 & 171.0 & 204.2 & 291.4 & 61.9 \\
\hline 3 & Binary & Excess & $10 \%$ & One-stage & 173 & 172.7 & $172.7^{1}$ & 92.4 & 92.3 & 35.4 & 95.2 & 75.2 \\
\hline \multirow[t]{2}{*}{4} & Continuous & Relative & $5 \%$ & Exponential & 0.297 & 0.302 & 0.304 & 0.198 & 0.229 & 0.112 & 0.162 & 0.277 \\
\hline & & & & $\mathrm{Hill}^{23}$ & 0.297 & - & 0.309 & 0.198 & - & 0.159 & 0.189 & 0.287 \\
\hline \multirow[t]{2}{*}{5} & Binomial & Excess & $10 \%$ & Log-logistic & 3.2 & 3.2 & 3.2 & 1.84 & 1.84 & 1.63 & 2.1 & 1.42 \\
\hline & & & & Log-normal & 3.31 & 3.31 & 3.31 & 1.98 & 1.98 & 1.78 & 2.23 & 1.58 \\
\hline
\end{tabular}

${ }^{1}$ Starting values required to get the reported results

${ }^{2}$ Potential problem with PROAST reporting the same values for the Hill and the exponential model. In the EFSA report results for Hill were: $B M D=0.302$ and $B M D L=0.205$ 
${ }^{3}$ Not possible to fit a three-parameter Hill model for these data in BMDS. In bmd a three-parameter log-logistic model was used instead. 